\title{
The identification of the direction of electrocutaneous stimulation along lineal multistimulator arrays
}

\author{
DAVID W. SPARKS \\ Department of Speech and Hearing Sciences DE-12, University of Washington \\ Seattle, Washington 98195
}

\begin{abstract}
Three experiments were conducted in an effort to evaluate the ability of observers to identify the direction of brief-duration spatiotemporal patterns applied to the skin. In Experiment 1 , observers were required to identify the direction of linear sweeps of electrical stimulation having varying total durations (sweep durations). Performance was evaluated at several loci on the body. The effects of stimulator number and spacing on the identification of sweep direction were evaluated in Experiment 2. In Experiment 3, the effects of temporal sequencing of stimuli on the "apparent movement" phenomenon were evaluated at two body loci: the forearm and the abdomen. The results from all three experiments suggest that the ability to identify the direction of spatiotemporal sweeps can be heavily influenced by body locus, number, and spacing of electrodes. In short, the design of the tactile display may have important ramifications to the success of any sensory substitution transform hardware.
\end{abstract}

In the past decade, a renewed interest in the use of the tactile system as a sensory substitution channel has been generated. A variety of elegant hardware devices has been fabricated to aid the blind in both reading (Bliss, Katcher, Rogers, \& Shepard, 1970) and in the identification of objects in their visual fields (White, 1970). Other researchers have developed speech-to-tactile transform hardware to aid the hearing-impaired in the reception and production of spoken language (Engelmann \& Rosov, 1974; Goldstein \& Stark, 1976; Saunders, 1974; Sparks, Kuhl, Edmonds, \& Gray, 1978; Scott \& DeFilippo, Note 1).

In associated research efforts, considerable attention has been centered on evaluating the basic response patterns of the skin. Frequency and amplitude sensitivities to tactile stimuli have been determined using single stimulators (Békésy, 1959a; Craig, 1972, 1974; Franzen \& Nordmark, 1975; Goff, 1967; Green \& Craig, 1974). Measurements of tactile detection thresholds in the presence of maskers (Craig, 1974; Gescheider, Herman, \& Phillips, 1970; Gilson, 1969; Sherrick, 1964), measurements of temporal acuity (Békésy, 1959b; Gescheider, 1967, 1970; Schmid, 1961), temporal order (Hirsh \& Sherrick, 1961; Sherrick, 1970), and measurement of apparent movement (Alles, 1970; Geldard \& Sherrick, 1972; Kirman, 1974; Sherrick \& Rogers, 1966) have often been made using two stimulators or a single row of several stimulators (Kirman, 1975). There are also reports describing the psychophysical testing of tactile displays having a large number of stimulators in more complex arrangements (Bliss, Crane, Mansfield, \& Townsend, 1966; Geldard \& Sherrick, 1965, 1972; Rogers, 1970; Seeley \& Bliss, 1966; Geldard \& Sherrick, Note 2, Note 3, Note 4). Much remains to be done, however, in the assessment of tactile perception using complex, multistimulator displays.

Specifically, the effects of tactile display configuration and number and placement of electrodes on an observer's identification of the direction of a progression of pulse stimuli are of interest to those attempting to use the skin as a sensory substitute to impaired vision or hearing. It is the objective of this report to describe experiments investigating such interactions using two electrocutaneous displays: one is a single line of eight electrodes and the second consists of 288 electrodes arranged in an 8 by 36 matrix.

\section{EXPERIMENT 1}

It was the purpose of this experiment to evaluate the ability of human observers to discriminate differences in the direction of a spatiotemporal sweep of stimulation along a linear electrode display surface as a function of the total duration of the sweep. It was a further objective to determine if the locus of stimulation on the body surface affected the accuracy of the observer in identifying direction. Since rapidly changing stimulus patterns are important to perception in other sensory modalities, particularly audi- 
tory, it seems reasonable to determine the response limitations of the tactile system to spatiotemporally varying stimulus patterns.

\section{Procedure}

Two young adults who were well trained (15 daily practice sessions, $45 \mathrm{~min}$ in duration) in the experimental tasks were employed as observers. Each observer wore one of two possible electrocutaneous displays. One display was comprised of a single row of eight electrodes spaced at $1.9 \mathrm{~cm}$ on center for a total length of $15.2 \mathrm{~cm}$. The second display, designed to transmit speech through the tactile modality (Sparks et al., 1978) was comprised of 288 electrodes (8 rows by 36 columns) spaced $1.3 \mathrm{~cm}$ on center. This larger display was approximately $10.2 \mathrm{~cm}$ wide, $45.7 \mathrm{~cm}$ long, and encircled the abdomen of the user. Figure 1 is a schematic of the configurations for both displays.

The observers wore the small display on two surfaces: on the volar surface of the forearm and on the abdomen. The larger display was worn only on the abdomen. The small display, designed with a foam rubber backing to assure contact between the electrodes and the skin, was secured with Velcro straps to the volar surface of the forearm and secured to the abdomen in both a horizontal direction and a vertical direction. In both cases of abdominal placement, the display was situated so that electrocutaneous stimulators never crossed the midline. The large display was secured around the abdomen using an elastic girdle arrangement that was backed by foam rubber and fastened by Velcro straps. The electrodes for all displays were designed by Saunders (1974) and were concentric. The active electrode was a silverplated disk with a radius of $2.5 \mathrm{~mm}$ and an area of $19.6 \mathrm{~mm}^{2}$ and was surrounded by an insulating ring and a silver-plated ground plate having an area of $107 \mathrm{~mm}^{2}$.

Both tactile displays were driven by a pair of independently programmable pulse generators. Two pulse generators were required to provide the capability of presenting two different pulse-burst sequences in rapid succession for psychological testing using a two-alternative forced-choice (2AFC) paradigm. In order to achieve pulse-burst sequences having identical timing but moving in opposite directions along either display, one system was slaved to the other.

The stimuli used in the experiments discussed throughout this report consisted of bipolar current pulses that were gated into pulse bursts having individual durations of $.45 \mathrm{msec}$. Four bipolar pulses made up each pulse burst, and pulses were current limited to $8 \mathrm{~mA}$ peak having zero net current flow. The pulse widths of the individual positive and negative pulses were always equal and could be varied between 5 and $25 \mu \mathrm{sec}$ with a time separation between them equal to $50 \mu \mathrm{sec}$ minus the pulse width. The pulse repetition rate was 10,000 pps.

Observers were required to attend to two observation intervals,
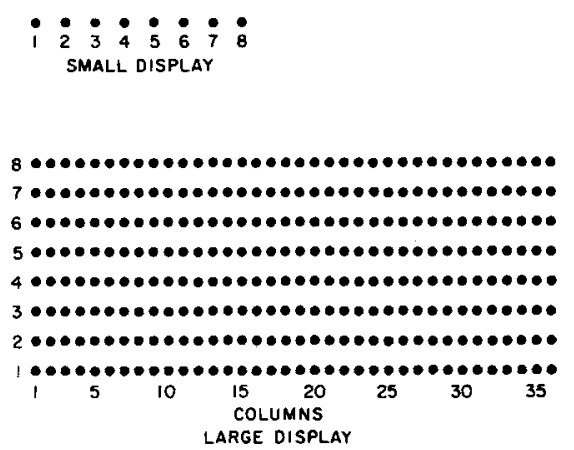

Figure 1. Schematic illustrating the electrode configurations for the small display and the large display. within which either eight single electrodes or eight clusters of four electrodes connected in parallel (large display) were sequentially activated by pulse bursts. The stimulus train in each interval always consisted of eight pulse bursts, wherein the first pulse burst was sent to the first electrode or electrode column, the second pulse burst was sent to the second electrode or electrode column, and so on. Referring to Figure 1, the clusters of four electrodes, connected in parallel, were either the bottom four rows of odd Columns 1-15 on the large display (for sweeps in the horizontal direction), or Columns $1,3,5$, and 7 for Rows 1 through 8 (for sweeps in the vertical direction).

Interstimulus onset intervals (ISOIs), defined as the time between the onsets of pulse bursts, were variable, ranging from .51 to $5.65 \mathrm{msec}$. An ISOI of $.51 \mathrm{msec}$ produced a pulse-burst sequence that "swept" across the display in a total time of $4 \mathrm{msec}$. The 5.65-msec ISOI produced a sweep duration equivalent to $40 \mathrm{msec}$. Individual pulse widths were controlled by observers and were adjusted to set overall stimulus intensity to a comfortable level.'

In one observation interval, the pulse-burst sequences swept from one end of the display to the other, and in the other interval, the sweep progressed in the opposite direction.

After attending to both observation intervals, the observer was required to depress a button corresponding to the interval in which the sequence moving away from the elbow or away from the abdominal midline (depending on display placement) occurred. This sequence occurred in Interval 1 with probability .5. The observation intervals were separated by $1 \mathrm{sec}$, and the observers were given a 3-sec response interval. Feedback was provided after every trial.

A total of 10 sweep durations selected from the results of pilot testing was employed in Experiment 1. These durations ranged between 4 and $40 \mathrm{msec}(4.0,4.4,5.0,5.7,6.7,8.0,10.0,13.3$, 20.0 , and $40 \mathrm{msec}$ ) and were calculated as the total time from the onset of the first pulse to the offset of the final pulse. The 10 ISOIs corresponding to sweep durations ranging from 4 to $40 \mathrm{msec}$ were $.51, .56, .65, .75, .89,1.08,1.36,1.84,2.79$, and $5.65 \mathrm{msec}$.

There were a total of 30 experimental conditions: 10 sweep durations for both body placements of the small display and 10 sweep durations for the large display. These experimental conditions were administered randomly, with the restriction that once a condition had been selected, both observers made all of the experimental judgments for that condition. A total of 200 trials, 100 per observer, was administered for each experimental condition.

\section{Results and Discussion}

The results of this experiment, shown in Figure 2, are plotted as the average over 200 trials for both observers, reported in percent correct identification of the direction of stimulus sequence (sweep direction) vs. the duration of the sequence (sweep duration). Data were averaged since there was small betweenobserver variance. The parameter is display condition, that is, forearm and abdominal placement of the small display, and abdominal placement of the large display. Visual lines of "best fit" are drawn through the points plotted in Figure 2 .

Examination of the data shows that best identification performance was achieved for the forearm placement of the small display. This curve shows that performance (identification of the sweep progressing away from the elbow) increased with increases in sweep duration. Interpolation from the visually fit line indicates that $75 \%$ correct falls at a sweep duration of about $5 \mathrm{msec}$. 


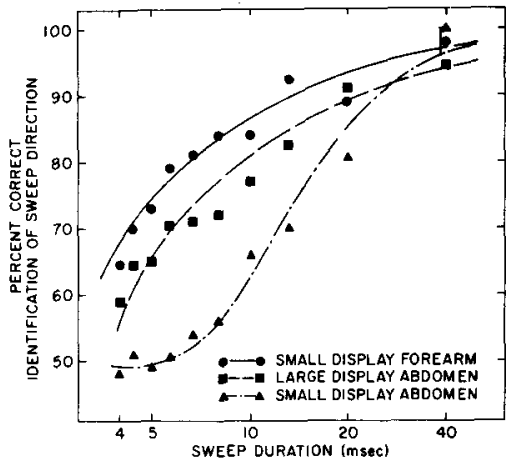

Figure 2. Mean percent correct identification of sweep direction (ordinate) for both observers as a function of sweep duration (abscissa). The parameter is display condition.

Contrast the results of the small display placement on the forearm with its placement on the abdomen. Identification of the sweep progressing away from the midline was $100 \%$ correct for the $40-\mathrm{msec}$ sweep duration, but there was a very precipitous falloff in performance as sweep duration was shortened. Interpolation from the visually fit line indicates that $75 \%$ correct falls at a sweep duration of about $14 \mathrm{msec}$. Subjective reports indicated that it was hard to establish a reliable identification cue for the abdominal placement of the small display. In fact, when the small display was placed on the abdomen, subjects complained that it was difficult to set stimulus intensity to a level of comfort. Adjustments of pulse width resulted in perceptual sweeps that often appeared either marginally detectable or too intense, but subjects were required to adjust stimulus magnitude until they arrived at the same level as they experienced with forearm placement.

This reduced dynamic range of senation magnitude is reminiscent of the sensory recruitment demonstrated with a receptor surface having low neural density (Békésy, 1959b). It is not clear that subjects were experiencing recruitment with the small display, but no experience of reduced dynamic range was ever reported by subjects undergoing speech testing with the large display similarly placed on the abdomen (Sparks et al., 1978). The large display, of course, has more active electrodes and undoubtedly stimulates more cutaneous receptors.

Pulse-burst sequences, identical to those employed with the smaller display, were delivered to every other column on the large display. Columns consisted of four electrodes. Although the length of the activated display surface was similar between small (approximately $15-\mathrm{cm}$ ) and large (approximately $19-\mathrm{cm}$ ) displays, it was posited that a greater number of receptors on the abdomen would be stimulated with the latter arrangement as a function of the increase from 8 to 32 in the number of active electrodes. The experimental task was the same as for the abdominal placement of the small display, and subjects reported no difficulty in setting stimulus intensity to the same level of comfort as used with the small display.

Figure 2 indicates that performance with the large display was slightly inferior to the forearm placement but superior to the abdominal placement of the small display. Interpolating from the visually fit line of this function, the $75 \%$ correct point falls at about $7 \mathrm{msec}$. This result, taken in relation to the result obtained for the abdominal placement of the small display, may imply that as more stimulators (and perhaps more receptor-neural units) become involved in the encoding of stimulus sweeps, better identification of stimulus direction results.

Finally, the identification of sweep direction in the vertical plane of the abdomen was tested for both small and large displays. Because stimulus parameters may be coded as spatiotemporal sweeps in the vertical plane for some visual or auditory-to-tactile transforms (for example, amplitude on a speech aid), the vertical plane was employed in this experiment. It is well known (Ruch, 1965) that the neural innervation to the abdomen courses horizontally from the spinal cord around to the abdominal midline. Therefore, it is probable that horizontal stimulus sweeps result in within-dermatome neural patterns and vertical sweeps result in across-dermatome neural patterns. Nonetheless, performance for both observers in the vertical plane was almost identical to performance in the horizontal plane for both displays.

In summary, two findings emerging from this experiment are of interest. First, the ability of these observers to identify the direction of stimulus sweeps having durations as short as $5 \mathrm{msec}$ should be encouraging to sensory-aid researchers, inasmuch as the skin has long been considered as "slow" with respect to its utility as a sensory substitute (Kirman, 1973). It may be that the encoding of such brief events as those often found in speech, for example, can be optimized for perception through the use of a multistimulator display.

Second, both the locus of stimulation on the body and the display format appear to have an effect on an observer's performance. Comparison between forearm and abdominal placements of the small display in Experiment 1 indicate that body locus affects the identification of sweep direction, at least when the number of electrodes was held constant between the two placements. Comparisons between performance for the abdominal placements of small and large displays imply that the number of electrodes on a display surface may also have an effect on the perception of direction.

\section{EXPERIMENT 2}

Experiment 1 led to the hypothesis that the identification of stimulus direction may be strongly 
influenced by the number and spacing of stimulators in a given display. In this experiment, the intention was to directly test the hypothesis that display number and spacing of electrodes may have a role in the determination of directional identification by a human observer.

\section{Procedure}

The experimental apparatus was identical to Experiment 1. The small display was employed only on the volar surface of the forearm, and the large display was placed on the abdomen. The number of electrodes on the small display was reduced from eight in two ways: It was reduced to four by activating every other electrode (Electrodes 1, 3, 5, and 7) and to two by activating the first and last electrodes (Electrodes 1 and 8 ). In this way, the display length was held constant at approximately the same length as that of the eight-electrode display employed in Experiment 1. The number of electrodes on the large display was reduced by activating every fourth column (Columns 1, 5, 9, and 13) while maintaining the $20-\mathrm{cm}$ display length employed in Experiment 1. The large display was also reduced to two active columns by activating the endpoints (Columns 1 and 15 in Figure 1).

Again, pulse-burst sweep durations between 4 and $40 \mathrm{msec}$ were generated. For example, in order to generate a pulse-burst sweep having a duration of $40 \mathrm{msec}$ for the four-electrode or fourcolumn display condition, pulse bursts were set such that they had 13.2-msec IOSIs. In the two-electrode or two-column condition, a 39.1-msec ISOI produced a 40-msec "sweep." The task of the observers was the same as in Experiment 1-that is, to identify the interval containing the stimulus sweep progressing away from the elbow (small display) or away from the midline (large display). The subjects attempted to set overall sensation magnitude to be equivalent for all experimental conditions, that is, irrespective of the number of activated stimulators.

\section{Results and Discussion}

Figures 3 and 4 show mean identification performance for both observers in percent correct vs. sweep duration obtained from the forearm placement of the small display and the abdominal placement of the large display, respectively. The parameter in both figures is the number of active electrodes for each display. The data plotted for eight electrodes were taken from Experiment 1. Visual lines of "best fit" are drawn through each function.

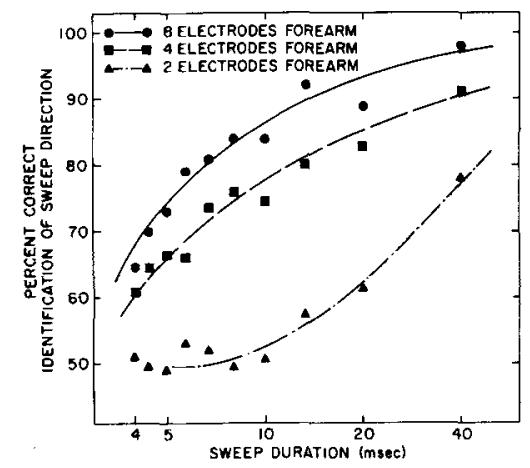

Figure 3. Mean percent correct identification of sweep direction along the forearm for both observers as a function of sweep duration. The parameter is the number of activated electrodes.

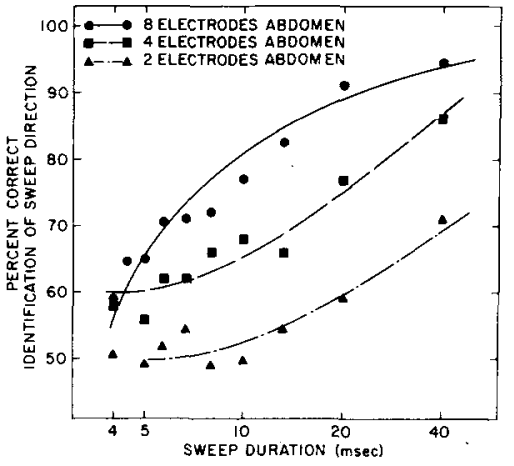

Figure 4. Mean percent correct identification of sweep direction along the abdomen for both observers as a function of sweep duration. The parameter is the number of activated electrodes.

The most noticeable feature in both figures is the progressive decline in performances as the number of active electrodes is decreased. The $75 \%$ correct points for the forearm placement of the small display (Figure 3) fall at about 5, 8, and $36 \mathrm{msec}$ for eight, four, and two active electrodes, respectively, and for the abdominal placement of the large display (Figure 4), fall at about 7, 20, and $58 \mathrm{msec}$ for eight, four, and two active columns, respectively.

Even though these results indicate that an observer's ability to detect the direction of stimulus progression can be influenced by the number of stimulators in a tactile display, the effect of stimulator spacing could not be determined. This is because the number of stimulators and spacing between stimulators were simultaneously varied, and both variables may have affected observers' performances. In the second half of this experiment, the number of electrodes was held constant while their spacing was changed. The large abdominal display provided a convenient means of altering stimulator spacing, without the requirement of making physical hardware changes.

Three display sizes were chosen: 10-, 20-, and 46- $\mathrm{cm}$ lengths along the long dimension of the large display. In the $10-\mathrm{cm}$ condition, eight adjacent columns of four electrodes separated by $1.3 \mathrm{~cm}$ (Columns 1 through 8 of Figure 1) were activated. In the $20-\mathrm{cm}$ condition, every other column between Columns 1 and 15 was activated. This resulted in columns being separated by $2.5 \mathrm{~cm}$ and was the same arrangement employed in Experiment 1. Finally, for the 46-cm condition, every fifth column was activated (Columns 1, 6, 11, 16, 21, 26, 31, 36, of Figure 1). In this condition, columns were separated by about $6 \mathrm{~cm}$. Stimulus sequences were generated in the same way as before. Only six sweep durations were employed: $5.0,5.7,6.7,8.0,10.0$, and $13.3 \mathrm{msec}$. The task of the observers was identical to that of Experiment 1.

Figure 5 is a plot of identification performance vs. 


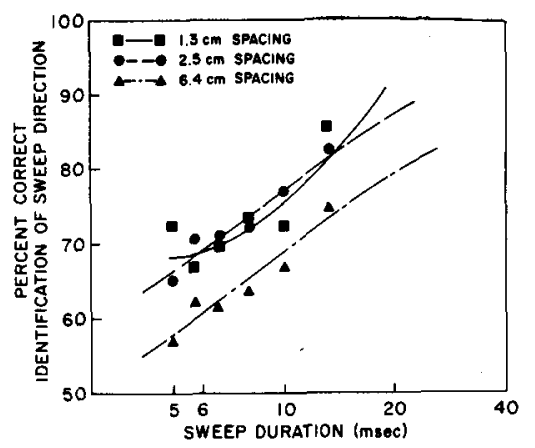

Figure 5. Mean percent correct identification of sweep direction along the abdomen of both observers as a function of sweep duration. The parameter is spacing between electrode columns.

sweep duration for the three conditions of electrode separation. Each point is a mean of 200 observation trials representing 100 from each observer; the data plotted for the $20-\mathrm{cm}$ display are taken from Figure 2. Visually fit lines are drawn through the data.

It is apparent from this figure that the $1.3-\mathrm{cm}$ spacing and $2.5-\mathrm{cm}$ spacing resulted in equivalent performance $\mathbf{~} 75 \%$ correct identification for sweep durations of about $9 \mathrm{msec}$ ). This result was interpreted as being a preliminary indication that, within certain limits, display size may be altered without greatly affecting the clarity of the directional information, so long as the number of stimulators is held constant.

The reason for the somewhat poorer performance seen when electrodes were spaced by $6 \mathrm{~cm}$ is not readily apparent. However, part of the reason may be that when stimulators were spaced by $6 \mathrm{~cm}$, stimulus sequences crossed the abdominal midline. Geldard and Sherrick (Note 3) have indicated that cross-midline placement of stimulators can deleteriously affect the kind of neural interactions that lead to the "rabbit" phenomenon, and there is little reason to doubt that the tracking of more rapid stimulus sweeps might be similarly affected.

Irrespective of the neural circuitry underlying these experimental observations, the point to be emphasized is that display format appears to be important to the perception of the spatiotemporal direction of pulseburst sequences. Kirman (1973) has pointed out that scientists involved in the past development of tactile communication aids have attempted to reduce the effects of simultaneous stimulus masking by separating electrodes over the surface of the body. This strategy may be costly, since the user may not be capable of perceptually integrating complex patterns under conditions of maximum electrode separation.

\section{EXPERIMENT 3}

This experiment was conducted in order to investigate the apparent movement phenomenon (Kirman, 1974, 1975; Sherrick \& Rogers, 1966) across the two displays used in Experiments 1 and 2. Two factors motivated this experiment. First, our laboratory was not aware of other sweep-direction experiments to which the findings in Experiments 1 and 2 could be directly compared. Second, Kirman's (1974) argument that the apparent movement phenomenon is relevant "to the spatiotemporal integration in tactile displays of speech" seems valid.

The objective of this experiment was to determine if the temporal sequencing of stimuli for apparent tactile movement across the eight-point displays differed significantly from that obtained with twopoint and four-point stimulator schemes reported by others.

In Experiments 1 and 2, the perception of apparent movement was not a requirement for performance. In fact, at the shorter sweep durations, observers described the percept as though someone was "pushing a finger into their forearm." The identification cue employed by observers was one of locus, not motion, wherein the "push" felt closer to the wrist or elbow, depending on sweep direction.

In Experiment 3, observers varied the repetition rate of the pulse bursts, determining sweep duration in such a way as to bracket good apparent movement. ${ }^{2}$

\section{Procedure}

The instrumentation and display hardware in this experiment were identical to those in Experiment 1. Pulse-burst durations were also identical $(.45 \mathrm{msec})$. However, observers used a method of adjustment to set the ISOI in such a way as to bracket good apparent movement. The means by which ISOI was varied, while holding pulse-burst width at a constant $.45 \mathrm{msec}$, was to vary the interpulse-burst interval. Decreasing the ISOI decreased the interpulse-burst interval and decreased the duration of the stimulus sweep from one display endpoint to the other.

Observers were familiarized with the distinctions between (1) stimulus successiveness appearing as "jumps" from electrode to electrode, (2) good apparent movement appearing as a smooth, continuous transition from one display endpoint to the other, and (3) movement appearing as though it were located more toward one end of the display than the other. This last percept will be termed "partial movement."

The observers were required to make two kinds of adjustments on a 10-turn potentiometer that continuously varied the ISOI. For one adjustment, they were required to set the potentiometer to that transition point at which their sensation just changed from stimulus successiveness to good movement. In the other adjustment, the task was to set the potentiometer to that transition point at which the sensation just changed from partial movement to good movement. The range of ISOI values covered by the 10 turns available on the potentiometer was between 3.3 and $100 \mathrm{msec}$.

Only the forearm placement of the small display (eight electrodes) and the abdominal placement of the large display (eight electrode columns) were used. Pulse-burst sweeps were accomplished in two ways. In one case, single electrodes or single columns of electrodes were sequentially activated by single-pulse bursts. In the other case, ISOI values were reduced by approximately half, and single electrodes or columns of electrodes were activated by two pulse bursts per stimulator location. Using this scheme, it was possible to hold total sweep duration constant while varying ISOI. Figure 6 illustrates the two types of stimulus sequences for a total sweep duration of $160 \mathrm{msec}$ across the small display. Observers were instructed to adjust stimulus magnitude 


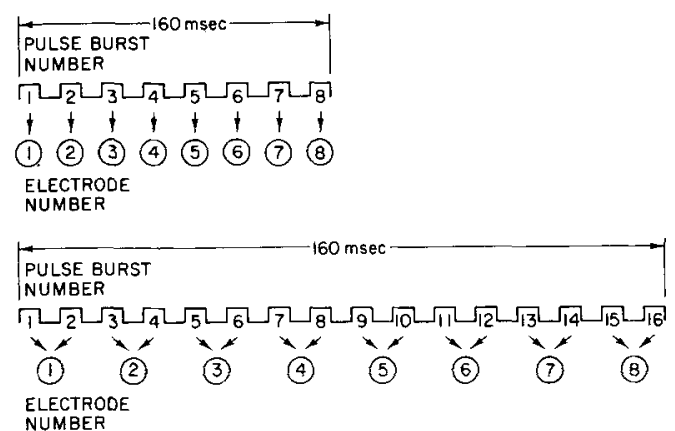

Figure 6. Illustration of the two types of stimulus sequences used in Experiment 3. The top sequence is referred to as the "single-pulse"' sequence, and the bottom sequence is the "doublepulse" sequence. The sweep duration is the same for both sequences (160 msec).

to the same level of comfort as experienced in Experiments 1 and 2. Each subject made 100 adjustments for each transition endpoint, resulting in a total of 200 adjustments for each transition endpoint at each body location.

\section{Results and Discussion}

The results from Experiment 3 are listed in Table 1. The means of the judgments at the transition points are given in terms of ISOI (milliseconds) and sweep duration (milliseconds) for both single- and doublepulse-burst stimuli (Figure 6) on the forearm and on the abdomen.

First, notice that forearm and abdominal displays rendered approximately the same results. This is not surprising, since for longer sweep durations (20 and $40 \mathrm{msec}$ ), performance in Experiment 1 was similar for the small display on the forearm and the large display on the abdomen.

Second, even though ISOI was approximately halved when the change from single- to double-pulseburst stimulation was made, the transition points for single- and double-pulse-burst stimulation fell at the same point when expressed in terms of sweep duration. Even though there were large ISOI differences between the two forms of pulse-burst sequences, the interelectrode offset intervals (IEOIs), defined as the time between the onset of the first burst to a given electrode and the onset of the first burst delivered to the adjacent electrode, were equivalent. The fact that equivalent IEOIs for single- and double-pulseburst sequences result in similar sweep-duration endpoints in the apparent movement continuum may imply that there is an integration time for movement associated with the skin. That is, when an electrode in an array is activated, there may be some time period (IEOI) over which multiple pulse bursts are integrated to yield the same sensation of movement as single pulse bursts having longer ISOIs.

One other point bears mentioning, since it may suggest an important difference between displays having a small number of electrodes and those with a larger number. If one assumes that the transition points shown in Table 1 represent endpoints on the stimulus continuum extending from successive movement to partial movement, one could suppose that good apparent movement is experienced in between. ${ }^{3}$ Although this notion was not formally tested, observers were asked to make 25 adjustments of the ISOI adjustment potentiometer in such a way as to slowly sweep across the entire continuum between the endpoints shown in Table 1 . They were asked to take careful notice of the nature of the movement sensations accompanying the continuously changing ISOIs between the endpoints described in Table 1, that is, for ISOIs ranging from approximately $26 \mathrm{msec}$ down to approximately $8 \mathrm{msec}$. Both observers independently agreed that they experienced good image movement between these endpoints.

There is a fairly substantial disagreement in the literature concerning the ISOI values leading to good apparent movement. For example, Sherrick and Rogers (1966) indicate that their observers required ISOIs of approximately $100 \mathrm{msec}$ between $3-\mathrm{msec}$ stimulus durations for good apparent movement between two vibrators. Kirman (1975) reports that his subjects had great difficulty experiencing good apparent movement for 1 - and 10-msec stimulus durations even when ISOIs were varied between 30 and $100 \mathrm{msec}$. In fact, his subjects required longer duration stimuli (50 and $100 \mathrm{msec}$ ) to achieve good apparent movement.

Table 1

Results from Experiment 3 Showing Transition Points from Successive Movement to Good Movement and from Good Movement to Partial Movement

\begin{tabular}{|c|c|c|c|c|c|c|}
\hline & & & \multicolumn{2}{|c|}{ Single-Pulse } & \multicolumn{2}{|c|}{ Double-Pulse } \\
\hline & & & Forearm & Abdomen & Forearm & Abdomen \\
\hline Successive $\rightarrow$ Good Movement & $\overline{\mathbf{x}}$ & $\begin{array}{l}\text { ISOI } \\
\text { Sweep Duration }\end{array}$ & $\begin{array}{r}25.6 \\
205.1\end{array}$ & $\begin{array}{r}27.8 \\
222.2\end{array}$ & $\begin{array}{r}13.3 \\
213.3\end{array}$ & $\begin{array}{r}12.3 \\
197.5\end{array}$ \\
\hline Good Movement $\rightarrow$ Partial Movement & $\overline{\mathbf{x}}$ & $\begin{array}{l}\text { ISOI } \\
\text { Sweep Duration }\end{array}$ & $\begin{array}{r}8.5 \\
67.8\end{array}$ & $\begin{array}{r}8.0 \\
66.7\end{array}$ & $\begin{array}{r}4.3 \\
69.0\end{array}$ & $\begin{array}{r}4.0 \\
63.7\end{array}$ \\
\hline
\end{tabular}

Note-Transition points are expressed in terms of ISOI and total sweep duration (in milliseconds) at both forearm and abdominal locations for single-and double-pulse stimulus sequences. 
The data from Experiment 3 suggest that stimuli having very brief durations $(.45 \mathrm{msec})$ and having ISOIs (or IEOIs) between as little as 8 to $26 \mathrm{msec}$ can lead to good apparent movement. We cannot explain the difference between these data and those reported by others. It may be that differences between subjects' interpretations of good apparent movement account for discrepancies between reports. Alternatively, it may be that subjects in Experiment 3 achieved good movement for short ISOIs because a greater number of stimulators were used here than were by other investigators. Thus, while individual stimulus durations and ISOIs were shorter, the total sweep times were longer, due to the greater number of stimulators. Kirman's (1975) experiment comparing apparent movement between two- and four-stimulator displays supports this latter hypothesis.

\section{GENERAL DISCUSSION}

The results from these experiments may be helpful to tactile sensory aid researchers in two ways: First, they provide some basic information relating to the information handling capacity of the tactile system, and second, they may provide some general guidelines for the design of either visual or auditory-to-tactile transforms. For example, Experiment 1 shows that an observer can identify some aspect of the direction of a stimulus sweep across an array of electrodes only so long as the duration of the sweep exceeds a value of approximately 5-10 msec. Further, it appears that the observer's performance can be maximized by appropriate display format and body placement.

Consider the application of these results to the perception of speech through an auditory-to-tactile transform. Speech perception researchers have taught us that the detection of the extent, direction, and tempo of rapid-frequency changes (formant transitions) is critical to speech-sound processing. These rapid transitions have typical durations on the order of $30-80 \mathrm{msec}$ and are important in the $F_{1}$ region of the spectrum (Stevens \& Klatt, 1974) as well as in the $F_{2}$ and $F_{3}$ regions (Fant, 1973; Liberman, Cooper, Shankweiler, \& Studdert-Kennedy, 1967; Stevens, 1975; Stevens \& Blumstein, 1976).

Spectrally oriented tactile speech aids (Sparks et al., 1978; Yeni-Komshian \& Goldstein, 1977) display the critical acoustic cues to speech perception in a way that may be related to the patterns described in Experiments 1 through 3. Since frequency and amplitude are coded in space on these aids, frequency transitions and intensity changes are coded as spatiotemporal patterns, that is, stimulus sweeps across an array of stimulators. The perception of speech sounds transformed by a tactile device, for example, their frequency transitions and amplitude changes into neighboring vowels, must then be limited by the temporal resolving properties of the skin. While an interpretation from the results of these experiments is limited by the fact that speech stimuli were not employed, the results do lead to the speculation that the perception of the basic properties of complex stimuli might be optimized if they were properly displayed in terms of number of stimulators (corresponding to frequency channels or amplitude levels), spacing of stimulators, and body locus of stimulation. This hypothesis should be tested by repeating aspects of Experiments 1 through 3 using synthetic speech tokens.

\section{REFERENCE NOTES}

1. Scott, B. L., \& DeFilippo, C. L. Evaluating a two-channel lipreading aid. Paper presented at the 92 nd Meeting of the Acoustical Society of America, San Diego, November 1976.

2. Geldard, F. A., \& Sherrick, C. E. Princeton cutaneous research project, Report No. 19, March 1972.

3. Geldard, F. A., \& Sherrick, C. E. Princeton cutaneous research project, Report No. 21, March 1973.

4. Geldard, F. A., \& Sherrick, C. E. Princeton cutaneous research project, Report No. 32, March 1974.

\section{REFERENCES}

Alles, D. S. Information transmission by phantom sensations. IEEE Transactions on Man-Machine Systems (MMS-11), 1970, 85-91.

BÉkÉsy, G. voN. Synchronism of neural discharges and their demultiplication in pitch perception on the skin and in hearing. Journal of the Acoustical Society of America, 1959, 31, 338-349. (a)

BÉkÉsY, G. von. Similarities between hearing and skin sensations. Psychological Review, 1959, 66, 1-22. (b)

Bliss, J. C., Crane, H. D., Mansfield, P. K., \& Townsend, J. T. Information available in brief tactile presentations. Perception \& Psychophysics, 1966, 1, 273-283.

Buiss, J. C., Katcher, M. H., Rogers, C. H., \& Shepard, R. P. Optical-to-tactile image conversion for the blind. IEEE Transactions on Man-Machine Systems (MMS-11), 1970, 58-65.

CRAIG, J. C. Difference threshold for intensity of tactile stimuli. Perception \& Psychophysics, 1972, 11, 150-152.

CraIG, J. C. Vibrotactile difference thresholds for intensity and the effect of a masking stimulus. Perception \& Psychophysics, 1974, 15, 123-127.

EngelmanN, S., \& Rosov, R. J. Tactual hearing experiment with deaf and hearing subjects. Oregon Institute of Research Bulletin, 1974, 14, 1-43.

Fant, G. C. Speech sounds and features. Cambridge, Mass: M.I.T. Press, 1973.

FRANZEN, O., \& NoRDMaRK, J. Vibrotactile frequency discrimination. Perception \& Psychophysics, 1975, 17, 480-484.

Geldard, F. A., \& SherRick, C. E. Multiple cutaneous stimulation: The discrimination of vibratory patterns. Journal of the Acoustical Society of A merica, 1965, 37, 797-801.

Geldard, F. A., \& Sherrick, C. E. The cutaneous 'rabbit': A perceptual illusion. Science, 1972, 178, 178-179.

Gescheider, G. A. Auditory and cutaneous temporal resolution of successive brief stimuli. Journal of Experimental Psychology, 1967, 75, 570-572.

GESCHEIDER, G. A. Some comparisons between touch and hearing. IEEE Transactions on Man-Machine Systems (MMS-11), 1970, 28-35.

Gescheider, G. A., Herman, D. D., \& Phillips, J. N. Criterion 
shifts in measurement of tactile masking. Perception \& Psychophysics, 1970, 8, 433-436.

GiLson, R. D. Vibrotactile masking: Effects of multiple maskers. Perception \& Psychophysics, 1969, 5, 181-182.

GoFF, G. D. Differential discrimination of frequency of cutaneous mechanical vibration. Journal of Experimental Psychophysics, $1967,74,294-299$.

Goldstein, M. H., \& Stark, R. E. Modification of vocalizations of preschool deaf children by vibrotactile and visual displays. Journal of the Acoustical Society of America, 1976, 50, 1477-1481.

Green, B. G., \& Craig, J. C. The roles of vibration amplitude and static force in vibrotactile spatial summation. Perception \& Psychophysics, 1974, 16, 503-507.

Hirsh, I. J., \& Sherrick, C. E. Perceived order in different sene modalities. Joumal of Experimental Psychology, 1961, 62, 423-432.

KIRMaN, J. H. Tactile communication of speech: A review and analysis. Psychological Bulletin, 1973, 80, 54-74.

KIRman, J. H. Tactile apparent movement: The effects of interstimulus onset interval and stimulus duration. Perception \& Psychophysics, 1974, 15, 1-6.

KIrman, J. H. The effect of number of stimulators on the optimal interstimulus onset interval in tactile apparent movement. Perception \& Psychophysics, 1975, 17, 263-267.

Liberman, A. M., Cooper, F. S., Shaniweiler, D. P., \& Studdert-Kennedy, J. Perception of the speech code. Psychological Review, 1967, 74, 431-461.

RoGERs, D. H. Choice of stimulator frequency for tactile arrays. IEEE Transactions on Man-Machine Systems (MMS-11), 1970, 5-11.

Ruch, T. C. Neural basis of somatic sensation. T. C. Ruch, H. D. Patton, J. W. Woodbury, \& A. L. Towe (Eds.), Neurophysiology. Philadelphia: Saunders, 1965.

SAunders, F. A. Electrocutaneous displays. In F. A. Geldard (Ed.), Conference on vibrotactile communication. Austin: The Psychonomic Society, 1974.

Schmid, E. Temporal aspects of cutaneous interaction with twopoint electrical stimulation. Joumal of Experimental Psychology, $1961,61,400-409$.

SEeley, H. F., \& Bliss, J. C. Compensatory tracking with visual and tactile displays. IEEE Transactions on Human Factors in Electronics (HFE-7), 1966, 84-90.

SherRick, E. C. Effects of double simultaneous stimulation of the skin. American Joumal of Psychology, 1964, 77, 42-53.

SEERricK, C. E. Temporal ordering of events in haptic space. IEEE Transactions on Man-Machine Systems (MMS-11), 1970, 25-28.

Sherrick, C. E., \& Rogers, R. Apparent haptic movement. Perception \& Psychophysics, 1966, 1, 175-180.
SPARKs, D. W., KuHR, P. K., EDMonds, A. E., \& GraY, G. P. Investigating the MESA (Multipoint Electrotactile Speech Aid): The transmission of segmental features of speech. Journal of the Acoustical Society of America, 1978, 63, 246-257.

Stevens, $K$. N. The potential role of property detectors in the perception of consonants. In G. Fant \& M. A. Tatham (Eds.), Auditory analysis and perception of speech. London: Academic Press, 1975.

Stevens, K. N., \& Blumstein, S. E. Context independent properties and place of articulation in stop consonants. Jourmal of the Acoustical Society of America, 1976, Suppl. 1, 59, 540(A).

STEVENS, K. N., \& KLATT, D. H. Role of formant transitions in the voiced-voiceless distinction of stops. Joumal of the Acoustical Society of America, 1974, 55, 654-659.

Whrte, B. W. Perceptual findings with the vision-substitution system. IEEE Transactions on Man-Machine Systems (MMS-11), 1970, No. 1, 54-58.

YeNI-Komshian, G. H., \& GoldsteIN, M. H. Identification of speech sounds displayed on a vibrotactile vocoder. Joumal of the Acoustical Society of America, 1977, 62, 194-198.

\section{NOTES}

1. Subjects set the individual bipolar pulse widths by adjusting them to comfort between 5 and $25 \mu \mathrm{sec}$. The adjustment was made by setting a potentiometer controlling the pulse width to all electrodes, meaning that all electrodes received pulses that were identical in width and current. The subjects were asked to set puise widths such that they rendered approximately the same sensation magnitude for all conditions reported in these experiments.

2. The term "good apparent movement" is used because observers were asked to bracket that range of sweep durations yielding "good, clear, and continuous motion from one display endpoint to the other." No attempt was made to arrive at a single-sweep duration producing optimal apparent movement.

3. This assumption finds support from a report by Geldard and Sherrick (Note 2), where subjects experienced a "cutaneous rabbit" hopping along their skin. The stimulus conditions were five 2-msec pulses in a row delivered to each of three positions on the arm. These authors report that, "The 'hops' disappear and a continuous sweep is felt if the intertap time is as short as $25 \mathrm{msec}^{\prime \prime}$ (p. 10). Note the similarity between ISOI at the successiveto-good movement boundary reported by these authors $(25 \mathrm{msec})$ and the ISOI for the same boundary $(26 \mathrm{msec})$ obtained in Experiment 3.

(Received for publication August 5, 1978; revision accepted October 15, 1978.) 